\title{
Design and analysis of uptilted aerial ladder for fire truck format
}

\author{
Zhi-Jian TIAN ${ }^{1,2}$, Xiang-Qin HAN ${ }^{1}$, Lei XU ${ }^{1}$, Li-Juan YAN ${ }^{3}$, Chong-Yi WEI', Jie $\mathrm{LI}^{4}$, Zhi-Song HOU ${ }^{4}$ and Wen-Guang JIANG ${ }^{4, a}$ \\ ${ }^{1}$ XCMG Construction Machinery Co., Ltd, XCMG fire-fighting safety equipment Co., Ltd, Xuzhou, China \\ ${ }^{2}$ School of mechatronic engineering, China university of mining and technology, Xuzhou, China \\ ${ }^{3}$ XCMG state key laboratory of intelligent manufacturing of advanced construction machinery, Xuzhou, China \\ ${ }^{4}$ School of mechanical engineering, Yanshan university, Qinhuangdao, China
}

\begin{abstract}
Aerial ladder fire truck is a kind of fire-fighting truck which is used for rescue of people and goods from high altitude locations. The aerial ladders of the fire truck are typically nested U-shaped truss structures manufactured with thin-walled steel by welding. Compared with close-shaped truss structures, their stiffnesses are relatively low. This means that they are quite slender structures, and they will deflect quite significantly due to their own weight and the working loads applied. If the original design of the ladder is straight, then the ladder will deform to a 'fishing-rod' shape in the air. The consequent 'bent-down' shape of the deformed ladder causes significant inconvenience during rescuing operations. To deal with this issue, a practical solution to the problem is that the ladder is manufactured with an appropriate uptilted curved shape, and it could deform to an approximately straight ladder when it is under certain desired working condition. In this paper, the effectiveness of the curved ladder design proposed in this paper has been validated by both finite element analysis and experimental results.
\end{abstract}

\section{Introduction}

Aerial ladder fire truck is a special fire-fighting truck equipped with extendable ladder system used for firefighting and rescuing from high altitude locations such as skyscrapers. A typical structure of an aerial ladder fire truck is shown in Fig. 1. Its structure can be considered to be composed of two major parts: the undercarriage and the boarding system. The undercarriage mainly consists of car chassis and an outrigger system which is used to enhance the against-overturning ability of the whole truck during working. The boarding system consists of turntable and ladder system. The turntable is mounted onto the chassis using a slewing bearing. The extendable ladder system consists of a base ladder mounted on the turntable and five telescopic sub-ladders. They are Ushaped truss structures nested together (see Fig. 1). There are sliding tracks designed on the sub-ladders of the ladder system which allow the adjacent sub-ladders to slide smoothly on each other during extension/retraction operations. The ladder system is powered by two sets of separate wire rope driving systems in parallel to realize synchronous extension or retraction operations. There are working platform and fire-fighting equipment installed on the tip of the ladder system, i.e. the end of sixth ladder. The inclined angle of the ladder system is controlled by the variable amplitude hydraulic cylinder. The slewing bearing allows the turntable together with the ladder system mounted on it to rotate full $360^{\circ}$ horizontally and this is controlled by a hydraulic motor.

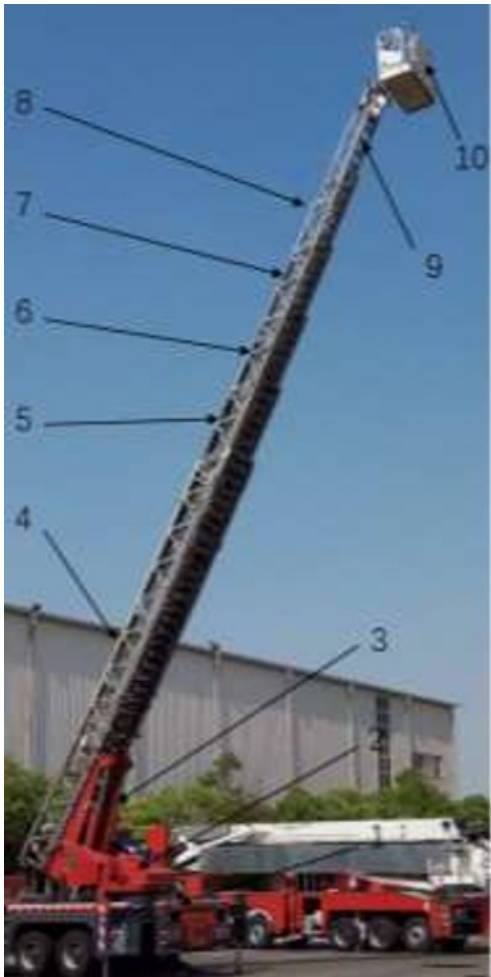

1.Car chassis; 2. turntable; 3 . variable amplitude hydraulic cylinder; 4. base ladder; 5 . second ladder; 6 . third ladder; 7 . fourth ladder; 8 . fifth ladder; 9. sixth ladder; 10 . working platform

Figure 1. Structure diagram of the aerial ladder fire-fighting truck

\footnotetext{
${ }^{a}$ Corresponding author: wgj@ysu.edu.cn
} 
In order to realize lightweight, the ladder system usually adopt the design of using U-shaped welded thinwalled truss structure [1]. When the ladder system is fully extended it is quite slender just like a 'fishing-rod'. From the point of view of both smooth operation of the machine and achieving clear vision for the operators, it is highly desirable that the fully extended ladder could stay in a straight-line configuration during working. A practical engineering solution to this problem is that the ladder system is designed with an uptilted curved original shape, the magnitude of the uptilt could just compensate the deflection caused by its self-weight and working loads, and as a consequence the ladder will show the desired straight configuration when loaded. To consider the importance of the smoothness of the extendingretracting operation process of the ladder system and easy-to-fabricate, the pre-bending ladder is preferably designed to have a single curvature circular arc shape. The aim of this paper is to develop numerical model to help decide the optimal radius for the pre-bending arc of the ladder system for a newly designed fire truck.

\section{Finite Element Model}

Due to the structural complexity of the aerial ladder, analytical solution to this mechanical system is very difficult to develop. In order to accurately predict the deformed shape of the complex aerial ladder system, finite element analysis has been employed [2]. To reduce the complexity of the establishment of the finite element mesh of the aerial ladder system with pre-bending curvature, we first build a finite element mesh of the straight ladder system, and then we use coordinate transformation to transform the finite element mesh of the straight ladder to the finite element mesh of the ladder system with a predefined pre-bending curvature. The transformation relation between the straight ladder and the pre-bending ladder is given by the following nodal coordinate transformation equations:

$x_{1}=\left(\mathrm{R}-y_{0}\right) \sin \left(x_{0} / \mathrm{R}\right)$

$y_{1}=R-\left(\mathrm{R}-y_{0}\right) \cos \left(x_{0} / \mathrm{R}\right)$

$z_{1}=z_{0}$

where $\mathrm{R}$ is the predefined radius for the uptitlted prebending arc shape of the ladder system, $\left(\mathrm{x}_{0}, \mathrm{y}_{0}, \mathrm{z}_{0}\right)$ are the nodal coordinates of the straight ladder, $\left(\mathrm{x}_{1}, \mathrm{y}_{1}, \mathrm{z}_{1}\right)$ are the nodal coordinates of the pre-bending ladder.

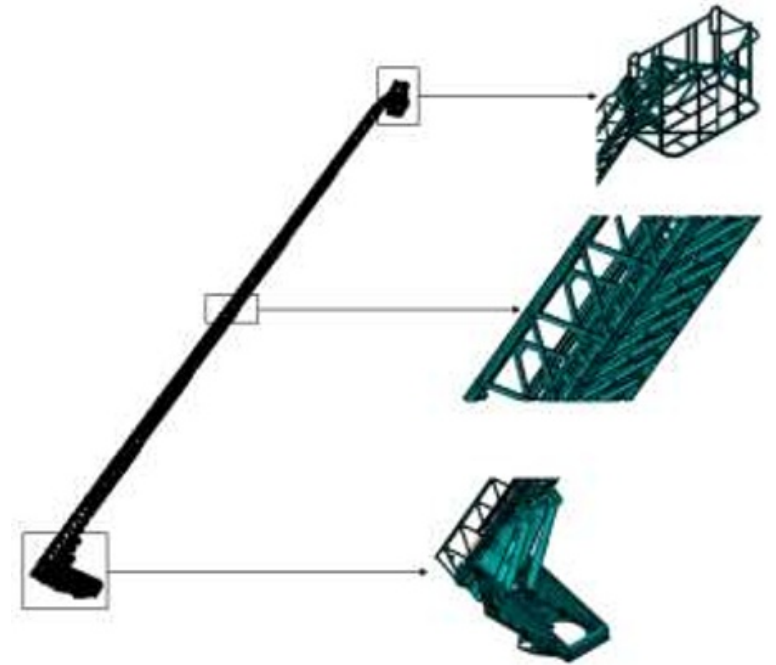

Figure 2. Finite element mesh of the straight aerial ladder.

Fig. 2 shows the finite element mesh of the straight ladder established. 8-noded solid brick elements have been used for the structural discretization. The total number of elements is 1029043 and the total number of nodes is 1718823 . Contact elements have been used to simulate the contact relationships between the neighbouring contacting sub-ladders. 2-noded link elements have used to simulate the rope systems driving the extension/extraction of the ladder systems. Fig. 3 illustrates the building process of the finite element mesh of the ladders with pre-bending from the already built finite element mesh of the straight ladder (see Fig. 2) using the transformation relationships of Eq. 1, Eq. 2, Eq. 3.

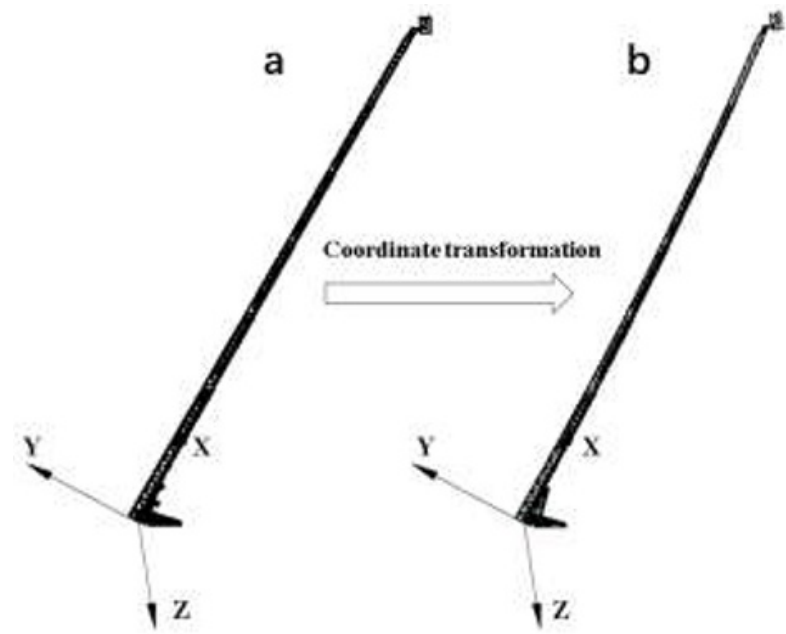

Figure 3. Illustration of the establishment process of the finite element mesh of the pre-bending ladder using coordinate transformation technique, (a) finite element mesh of an initially straight ladder, and (b) finite element mesh of the pre-bending ladder.

\section{Determination of the Optimal Pre- bending Radius and Experimental Verification}

The false position searching method [3] has been employed to find the optimal pre-bending radius of the ladder. It is an iterative numerical process. In each 
iterative step, finite element analysis has to be reperformed again, and from the nodal degree- of-freedom analysis result to obtain the extent of deviation from the ideal straight deformed shape. The iterative process continues until the deviation becomes negligibly small, i.e. the deviation of the inclined tip angle of the ladder is less than $0.1^{\circ}$. Due to the nature of large deformation of the structure, geometric nonlinear effect has been considered in the finite element analyses. Fig. 4 shows the deformed shape of the fully extended ladder superimposed with the undeformed configuration from the finite element analysis results from the ladder with the optimal radius. It can be seen from this figure that the pre-bending structure (shown as the configuration free from load in the figure) is obviously a curved shape, and it becomes a straight ladder (shown as the configuration after loading in the figure).

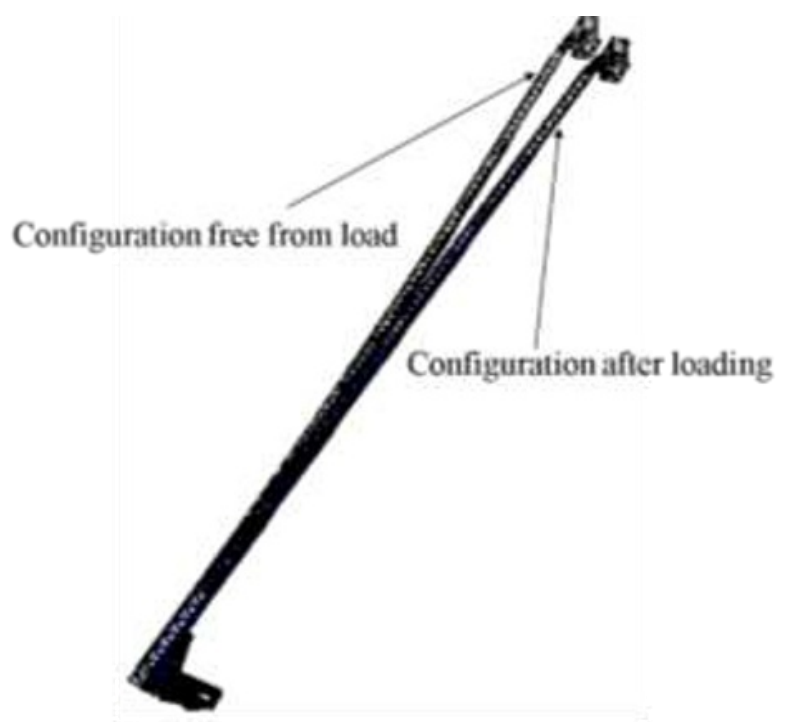

Figure 4. Deformed shape of the fully extended ladder superimposed with undeformed configuration

A real ladder using the predicted optimal radius obtained from this research has been manufactured, and a verification experimental test has been conducted on it. A specialized instrument called Focus X330 3D laser scanner (shown in Fig. 5) has been used to measure the deformed shape of the circular arc shaped aerial ladder with the predicted optimal bending radius. The measuring range of the laser scanner is $0.6 \mathrm{~m}-330 \mathrm{~m}$ and the measuring accuracy is $\pm 1 \mathrm{~mm}$, which is very accurate. The measurement process scanned and recorded spatial positions of all sample points which densely arranged along the whole length of the ladder system. Figure the location distribution of the sample points recorded which could represent the spatial shape of the extended aerial ladder under working load. In this figure, a reference straight line is also plotted as a baseline to show the effect of the uptilted arc shape design of the ladder system. The deformed shape of the ladder system can be seen to be very closely overlapped with the reference baseline, which indicates that Fig. 6 shows the prebending design is perfectly validated.

\section{Conclusion}

Finite element model for a pre-bending aerial ladder system for a newly designed fire truck has been developed. A numerical search process has been implemented to find the optimal radius of the pre-bending arc shape of the ladder system using the finite element model developed. The predicted optimum curved shaped aerial ladder has been manufactured. Verification experiment has been carried out on it. The optimal radius predicted in this paper has been validated by the experimental results.

\section{Acknowledgement}

The authors would like to acknowledge the financial support from National Key R\&D Program in China, Project title: Key Technology Research and Application Demonstration for High Mobility and Multi-Function Emergency Rescue Vehicle (funding number: 2016YFC0802900)

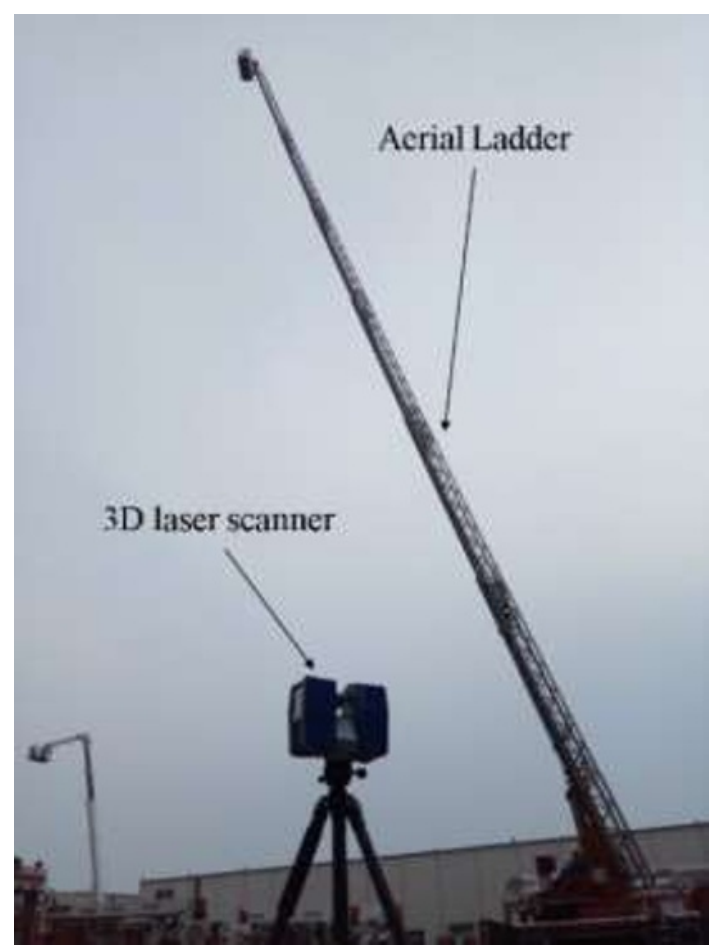

Figure 5. Photo showing a 3D laser scanner which is used to measure the deformed shape of the aerial ladder 


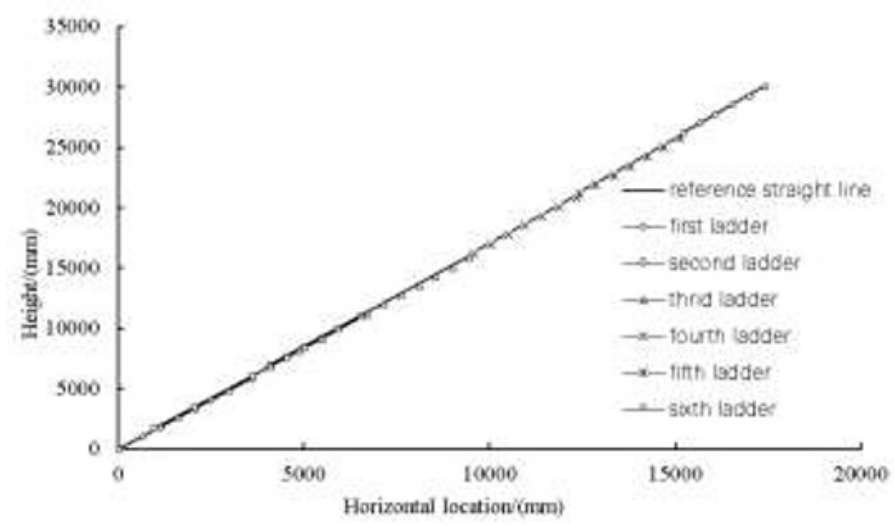

Figure 6. Experiment result showing that the deformed shape of the pre-bending ladder system is very close to an ideal straight baseline

\section{References}

1. Z. J. Tian et al. Design manual of aerial ladder truck, XCMG group, Internal document, (2017)

2. K. J. Bathe. Finite element procedures. Prentice Hall, (2006)

3. D. V. Griffiths and I. M. Smith. Numerical Method for Engineers. 2nd edition, Chapman \&. Hall/CRC press, (1991) 